\title{
Folliculostellate Cells Are Required for Laminin Release from Gonadotrophs in Rat Anterior Pituitary
}

\author{
Takehiro Tsukada ${ }^{1}$, Ken Fujiwara ${ }^{1}$, Kotaro Horiguchi ${ }^{3}$, Morio Azuma ${ }^{1}$, \\ Dini Ramadhani ${ }^{1}$, Alimuddin Tofrizal ${ }^{1}$, Khongorzul Batchuluun ${ }^{1}$, Rita Maliza $^{1}$, \\ Rahimi Syaidah ${ }^{1}$, Motoshi Kikuchi ${ }^{1,2}$ and Takashi Yashiro ${ }^{1}$ \\ ${ }^{I}$ Division of Histology and Cell Biology, Department of Anatomy, Jichi Medical University School of Medicine, Tochigi, Japan, \\ ${ }^{2}$ Laboratory of Natural History, Jichi Medical University School of Medicine, Tochigi, Japan and ${ }^{3}$ Laboratory of Anatomy and \\ Cell Biology, Department of Health Sciences, Kyorin University, Tokyo, Japan
}

Received June 18, 2014; accepted August 26, 2014; published online October 23, 2014

\begin{abstract}
The anterior pituitary gland is organized tissue comprising hormone-producing cells and folliculostellate (FS) cells. FS cells interconnect to form a meshwork, and their cytoplasmic processes are anchored by a basement membrane containing laminin. Recently, we developed a three-dimensional (3D) cell culture that reproduces this FS cell architecture. In this study of the novel function of FS cells, we used transgenic rats that express green fluorescent protein in FS cells for the 3D culture. Anterior pituitary cells were cultured with different proportions of FS cells $(0 \%, 5 \%, 10 \%$, and $20 \%)$. Anterior pituitary cells containing $5-20 \%$ FS cells formed round/oval cell aggregates, whereas amorphous cell aggregates were formed in the absence of FS cells. Interestingly, immunohistochemistry showed laminin-immunopositive cells instead of extracellular laminin deposition in FS cell-deficient cell aggregates. Double-immunostaining revealed that these laminin-immunopositive cells were gonadotrophs. Laminin mRNA expression did not differ in relation to the presence or absence of FS cells. When anterior pituitary cells with no FS cells were cultured with FS cellconditioned medium, the proportion of laminin-immunopositive cells was lower than in control. These results suggest that a humoral factor from FS cells is required for laminin release from gonadotrophs.
\end{abstract}

Key words: laminin, anterior pituitary, folliculostellate cell, gonadotroph, 3D cell culture

\section{Introduction}

The anterior pituitary gland consists of various cell types, including five types of hormone-producing cells and folliculostellate (FS) cells, which do not produce classical anterior pituitary hormones. These cells form clusters (socalled lobular structures), which are surrounded by extracellular matrix (ECM) $[17,18]$. The cells are not distributed randomly in each lobule; rather, hormone-producing cells have affinities for specific cell types (topographic affinity) [14]. FS cells are localized in the center of the lobule and

Correspondence to: Takashi Yashiro, M.D., Ph.D., Department of Anatomy, Jichi Medical University School of Medicine, 3311-1 Yakushiji, Shimotsuke, Tochigi 329-0498, Japan. E-mail: tyashiro@jichi.ac.jp form a meshwork structure by means of homotypical interconnection [18]. In addition, the cytoplasmic processes of FS cells attach to surrounding ECM and envelop hormoneproducing cells [12]. Histological characteristics suggest that local cell-cell and cell-ECM interactions are essential for the respective roles of anterior pituitary cells (for reviews see [3, 15]).

Our series of studies showed that laminin, a major basement membrane component, interacts with FS cells and influences FS cell functions $[9,10,19]$. Laminin is a heterotrimer of the $\alpha, \beta$, and $\gamma$ chains [20] and has various actions on cells through cell surface receptors such as the integrins and dystroglycans [22]. In the anterior pituitary gland, Holck et al. [6] detected immunoreactive laminin in the cytoplasm of gonadotrophs and in the vascular base- 
ment membrane. More than 20 years after the discovery of laminin in gonadotrophs, we used in situ hybridization to characterize laminin isoforms expressed in gonadotrophs and found that the laminin isoforms differed from those produced by vascular endothelial cells [16]. However, the mechanism of laminin synthesis and release in the anterior pituitary is not well understood. This report describes the novel action of FS cells on laminin secretion in gonadotrophs, which was discovered by means of a threedimensional (3D) cell culture of anterior pituitary cells from S100b-GFP transgenic rats, which express green fluorescent protein (GFP) in FS cells.

\section{Materials and Methods}

\section{Animals}

Wistar rats were purchased from Japan SLC (Shizuoka, Japan). Transgenic S100b-GFP rats [13] were kindly donated by Prof. K. Inoue of Saitama University and bred in our animal facility. Eight- to 10 -week-old male rats weighing 250-300 g were given ad libitum access to food and water and maintained under a 12-hr light/dark cycle. Room temperature was controlled at around $22^{\circ} \mathrm{C}$. All animal experiments were carried out in a humane manner after receiving approval from the Institutional Animal Experiment Committee of Jichi Medical University and were conducted in accordance with the Institutional Regulation of Animal Experiments and Fundamental Guidelines for Proper Conduct of Animal Experiments and Related Activities in Academic Research Institutions, under the jurisdiction of the Japanese Ministry of Education, Culture, Sports, Science and Technology.

\section{Hanging drop 3D cell culture}

Anterior pituitary cells were isolated from male S100b-GFP transgenic rats, as described previously [8]. Isolated cells were separated into GFP-positive cells (FS cells) and GFP-negative cells using a MoFlo XDP cell sorter (Beckman Coulter, Brea, CA, USA). GFP-positive and GFP-negative cells were mixed at a proportion of $0 \%$, $5 \%, 10 \%$, or $20 \%$ FS cells (a $5 \%$ proportion of FS cells is equivalent to that in normal adult rat anterior pituitary [4]). The hanging drop method was used for 3D culture, as described previously [21]. The cells were cultured for 5 days and processed for each experiment. We previously confirmed that an in vivo-like FS cell meshwork and filamentous laminin were formed 5 days after plating [21]. Cell aggregates were observed on an IX71 inverted fluorescence microscope (Olympus, Tokyo, Japan).

\section{FS cell-conditioned medium}

GFP-positive cells were collected as described above and plated onto 8 -well chamber slides $\left(1 \mathrm{~cm}^{2} /\right.$ well; Nalge Nunc International, Penfield, NY, USA) at a density of $1 \times 10^{5}$ cells $/ \mathrm{cm}^{2}$ in $400 \mu \mathrm{l}$ of medium. Three days after cell culture, the resulting medium was collected and stored at $-80^{\circ} \mathrm{C}$. On the day of the experiment, FS cell-conditioned medium was thawed and diluted to the appropriate concentration with fresh medium.

\section{Immunofluorescence microscopy}

Immunohistochemistry was performed as described previously [21]. Primary antibodies included rabbit polyclonal anti-mouse laminin (1:1600, LSL-LB-1013; Cosmo Bio, Tokyo, Japan), goat polyclonal anti-rat LH $\beta(2 \mu \mathrm{g} / \mathrm{ml}$, sc-7824; Santa Cruz Biotechnology, Santa Cruz, CA, USA) and anti-rat $\mathrm{GH}$ antibodies $(0.25 \mu \mathrm{g} / \mathrm{ml}, \mathrm{AF} 1566$; R\&D Systems, Minneapolis, MN, USA), and mouse monoclonal anti-human 17-39-ACTH $(0.625 \mu \mathrm{g} / \mathrm{ml}$, CBL56; Cymbus Biotechnology, Hampshire, UK), anti-human prolactin (0.625 $\mu \mathrm{g} / \mathrm{ml}$, 6F11; QED Bioscience, San Diego, CA, USA), and anti-human TSH $\beta(8 \mu \mathrm{g} / \mathrm{ml}, 506201$; Merck Millipore, Billerica, CA, USA) antibodies. For detection of vascular endothelial cells, biotinylated isolectin B4 (20 $\mu \mathrm{g} / \mathrm{ml}$, B-1205; Vector Laboratories, Burlingame, CA, USA), which binds to galactose residue on the surface of vascular endothelial cells [2], was added to the primary antibody. Secondary antibodies included Alexa Fluor 488conjugated goat anti-rabbit IgG (1:200), Alexa Fluor 568conjugated goat anti-rabbit IgG (1:200), Alexa Fluor 568conjugated donkey anti-goat IgG (1:200), and Alexa Fluor 568-conjugated goat anti-mouse IgG (1:200) (Life Technologies). Biotinylated isolectin B4 was detected by Alexa Fluor 568-conjugated streptavidin (1:400: Life Technologies). Coverslips were mounted onto cells using Vectashield mounting medium containing DAPI (Vector Laboratories, Burlingame, CA, USA). Stained cells were subsequently analyzed on a FV1000 confocal laser microscope (Olympus). Images were processed for presentation using Photoshop CS5 software (Adobe Systems, San Jose, CA, USA). For cell counting, aggregates stained for laminin were randomly picked and imaged with a 60-fold objective lens. Laminin-immunopositive cells were counted using Image J 1.43u software (National Institutes of Health, Bethesda, MD, USA). Data are presented as a percentage of the total number of DAPI-positive cells in each group $(n=5-10$, data are from 2-3 cultures).

\section{Quantification of $m R N A$ levels by real-time PCR}

One hundred to 150 cell aggregates were collected from 3D cell culture, and total RNA was extracted using an RNeasy mini-kit and RNase-free DNase set according to the manufacturer's instructions (Qiagen, Hilden, Germany). cDNA was synthesized using the PrimeScript RT reagent kit (Takara Bio, Otsu, Japan) with oligo-(dT) ${ }_{20}$ primer (Life Technologies). Quantitative real-time PCR (ABI PRISM 7900HT; Applied Biosystems, Foster City, CA, USA) was performed using gene-specific primers and SYBR Premix Ex Taq (Takara Bio) containing SYBR Green I $(n=4-5)$. Primers were designed for rat laminin $\alpha 1$ (LAMA1: NM 001108237.2): forward 5'-TTG ACC TCC ACG GAG TTT TC-3' and reverse 5'-ATG GGA ATG AAA GAC 
GCT TG-3' (product size: 133 bp). For internal calibration, mRNA transcribed from the standard gene encoding $\beta$-actin (NM_031144.3) was used: forward 5'-TGG CAC CAC ACT TTC TAC AAT GAG C-3' and reverse 5'-GGG TCA TCT TTT CAC GGT TGG-3' (product size: $106 \mathrm{bp}$ ).

\section{Statistical analysis}

All results are presented as mean \pm SEM. Unpaired two-tailed Student's $t$-test or Dunnett's test was performed using Prism version 6.0 (GraphPad Software, San Diego, CA, USA). A P value of $<0.05$ was considered to indicate statistical significance.

\section{Results}

\section{Effect of FS cells on laminin deposition}

Anterior pituitary cells were cultured in a hanging drop with FS cells $(5-20 \%)$ or without FS cells $(0 \%)$ for
5 days. In the presence of FS cells, cell aggregates had a round/oval shape with a smooth outer layer (Fig. 1b-d). In the absence of FS cells, however, the aggregates had an amorphous, irregular shape (Fig. 1a). Individual cells were identifiable at the fringe of these aggregates (Fig. 1a), which was not the case for aggregates containing FS cells (Fig. 1b-d). FS cells expressing GFP were interconnected homotypically by their cytoplasmic processes in the aggregates (Fig. 1f $-\mathrm{h}$ ). To examine the effect of FS cells on laminin deposition, we stained the aggregates for laminin. Filamentous and intermittently dot-like laminin staining was observed in the extracellular space when aggregates were cultured with FS cells (Fig. 1j, k, 1, n, o, p). Little or no laminin deposition was observed in the extracellular space of FS cell-deficient cell aggregates (Fig. 1i, m). Instead of extracellular laminin deposition, laminin-immunopositive cells were present at low concentrations of FS cells (Fig. 1i, j, m, n, arrowheads). As shown
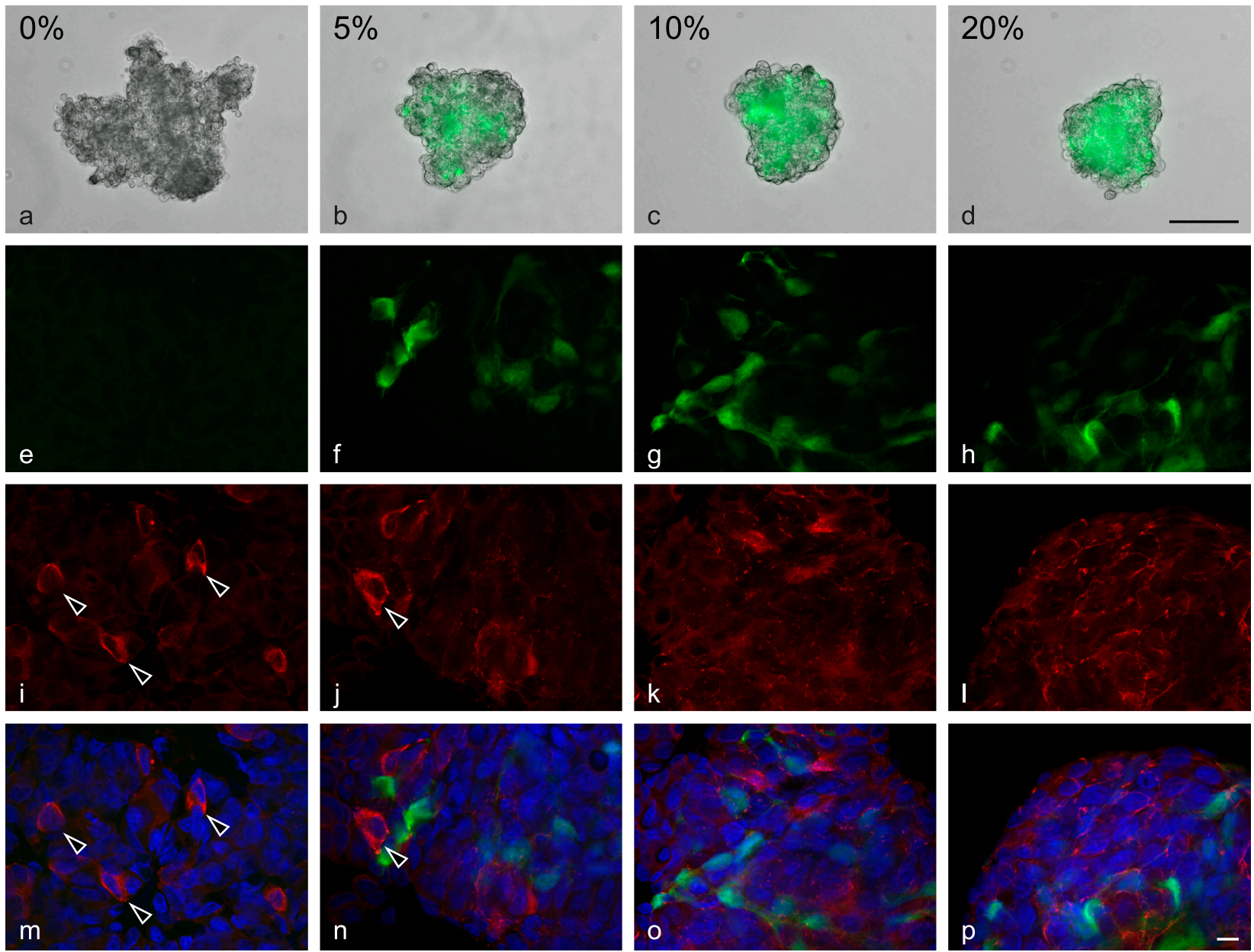

Fig. 1. Immunofluorescence of laminin in 3D cell aggregates with different proportions of FS cells $(0 \%-20 \%)$. Cell aggregates were fixed 5 days after plating and stained with laminin antibody. The top panels are phase-contrast images of cell aggregates superimposed on fluorescence images of FS cells (a-d). FS cells expressing GFP are shown in green. During the 5-day culture, amorphous aggregates were observed in the absence of FS cells (a). In the presence of FS cells, the cells formed round or oval aggregates with a smooth outer layer (b-d). The confocal images of FS cells and laminin immunofluorescence are shown in $\mathbf{e}-\mathbf{h}$ and $\mathbf{i}-\mathbf{l}$, respectively. The bottom panels show merged images (m-p; FS cells, green; laminin, red; DAPI, blue). Extracellular laminin deposition was observed as the number of FS cells increased (k, l, o, p), while laminin-immunopositive cells (arrowheads) decreased as the number of FS cells increased $(\mathbf{i}, \mathbf{j}, \mathbf{m}, \mathbf{n})$. Bars $=100 \mu \mathrm{m}(\mathbf{a}-\mathbf{d})$ and $10 \mu \mathrm{m}(\mathbf{e}-\mathbf{l})$. 


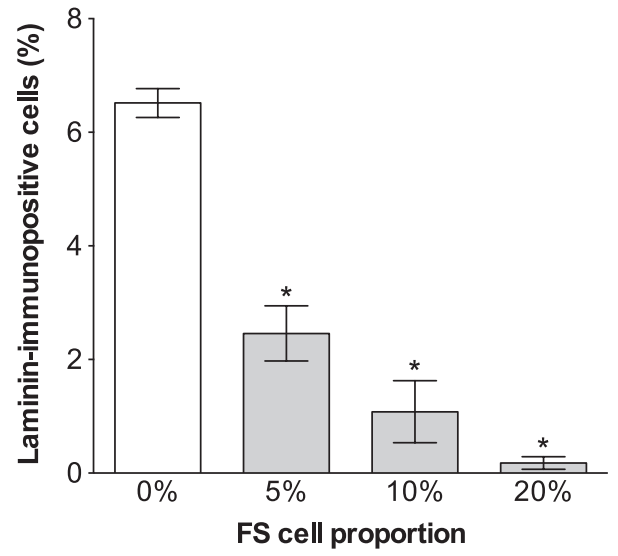

Fig. 2. Percentage of laminin-immunopositive cells in cell aggregates by the proportion of FS cells $(n=5-6$, mean \pm SEM). Cell aggregates were fixed 5 days after plating and stained with laminin antibody. The number of laminin-immunopositive cells was counted and normalized by the total number of cells (DAPI). Laminin-immunopositive cells decreased as the proportion of FS cells increased. The proportion of laminin-immunopositive cells in FS cell-deficient cell aggregates $(0 \%$ FS cells) significantly differed from that in cell aggregates containing $5 \%, 10 \%$, and $20 \%$ FS cells $\left({ }^{*} \mathrm{p}<0.05\right.$, Dunnett's test).

in Figure 2, the proportion of laminin-immunopositive cells decreased as the proportion of FS cells increased. The proportion in cell aggregates containing 5-20\% FS cells was significantly different from that in FS cell-deficient cell aggregates $\left({ }^{*} \mathrm{p}<0.05\right)$.

\section{Identification of laminin-immunopositive cells}

To identify laminin-immunopositive cells, FS celldeficient cell aggregates were stained for laminin and either a pituitary hormone or the vascular endothelial cell marker isolectin B4. Laminin-immunopositive cells stained for LH $\beta$ (Fig. 3a) but not for GH, ACTH, prolactin, TSH $\beta$, or isolectin B4 (Fig. 3b-f).

\section{Effect of FS cells on laminin synthesis and release}

Ramadhani et al. [16] found that only gonadotrophs produce laminin containing the $\alpha 1$ chain (Lamal) in the anterior pituitary of adult rats. Thus, we investigated the effect of FS cells on Lamal expression. mRNA expression of Lamal did not change in relation to the presence or absence of FS cells (Fig. 4). We next examined whether FS cells induce laminin release from gonadotrophs. FS celldeficient cell aggregates were cultured in media supplemented with $10 \%$ or $20 \%$ FS cell-conditioned medium for 5 days and stained for laminin (Fig. 5). Dot-like extracellular laminin deposition was observed when cultured with FS cell-conditioned medium (Fig. 5e, f, h, i, arrowheads). However, unlike cell aggregates containing FS cells (Fig. 1j, k, 1, n, o, p), FS cell-deficient aggregates cultured with FS cell-conditioned medium did not display filamentous laminin staining. The proportion of lamininimmunopositive cells was lower when cultured with FS cell-conditioned medium (Fig. 6). The proportion in cell aggregates cultured with normal media $(0 \%)$ differed
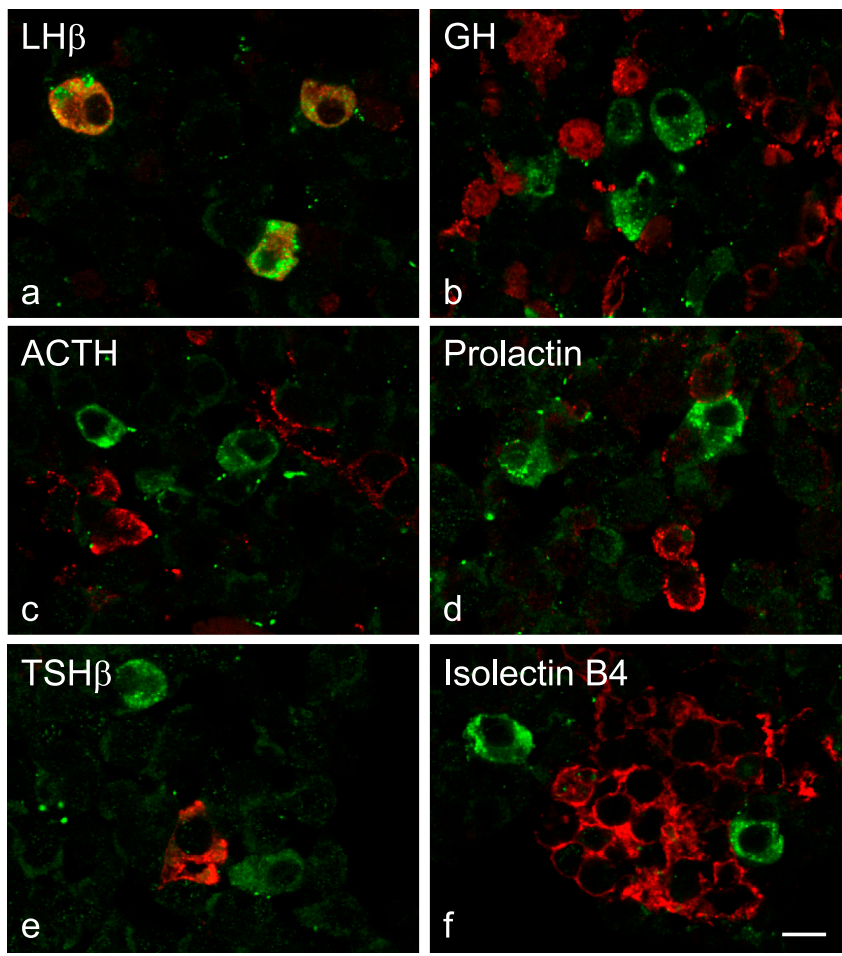

Fig. 3. Identification of laminin-immunopositive cells in FS celldeficient cell aggregates. Cell aggregates with no FS cells were fixed 5 days after plating and co-stained with laminin antibody and either a hormone antibody (a-e) or isolectin B4 to stain vascular endothelial cells (f). Laminin is shown in green. Hormones and isolectin B4 are shown in red. Laminin-immunopositive cells were co-localized only with $\operatorname{LH} \beta$ (a). Bar $=10 \mu \mathrm{m}$.

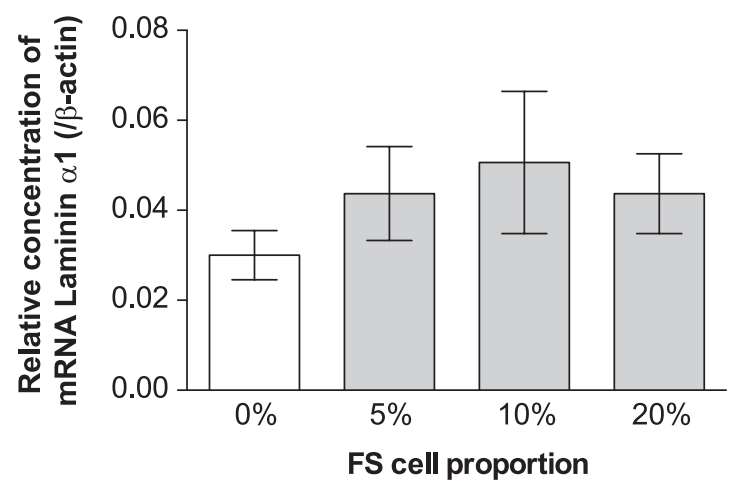

Fig. 4. Relative mRNA concentration of laminin $\alpha 1$ chain (Lamal), evaluated by quantitative real-time PCR. The white and gray bars represent cell aggregates without FS cells $(0 \%)$ and with FS cells $(5 \%, 10 \%$, and $20 \%$ ), respectively $(n=4-5$, mean \pm SEM). The concentrations were normalized with $\beta$-actin mRNA concentration. The expression level of Lamal mRNA did not significantly differ in relation to the presence or absence of FS cells.

significantly from those in cells cultured with FS cellconditioned medium. However, there was no significant difference between the proportions for $10 \%$ and $20 \%$ FS cell-conditioned media. 

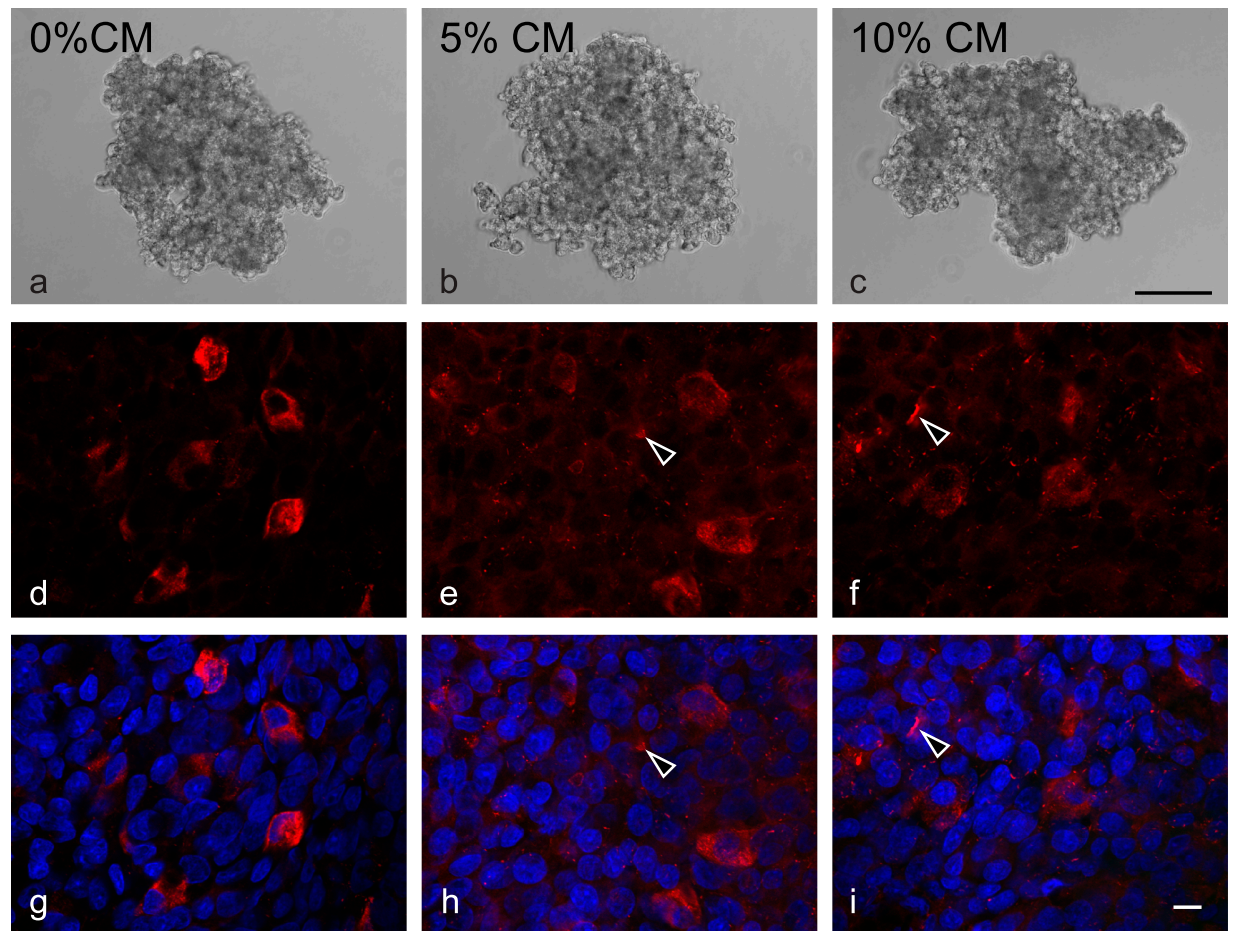

Fig. 5. Immunofluorescence of laminin in FS cell-deficient aggregates cultured with different concentrations of FS cell-conditioned medium (CM; $0 \%-$ $20 \%$ ). Cell aggregates were fixed 5 days after plating and stained with laminin antibody. The top panels show phase-contrast images of cell aggregates $(\mathbf{a}-\mathbf{c})$. The middle and bottom panels show confocal images of laminin immunofluorescence (d-f) and merged images (g-h; laminin, red; DAPI, blue), respectively. Dot-like extracellular laminin deposition (arrowheads) was observed in cell aggregates cultured with FS cell-conditioned medium (e, f, $\mathbf{h}$, i). Bars $=100 \mu \mathrm{m}(\mathbf{a}-\mathbf{c})$ and $10 \mu \mathrm{m}(\mathbf{d}-\mathbf{f})$.

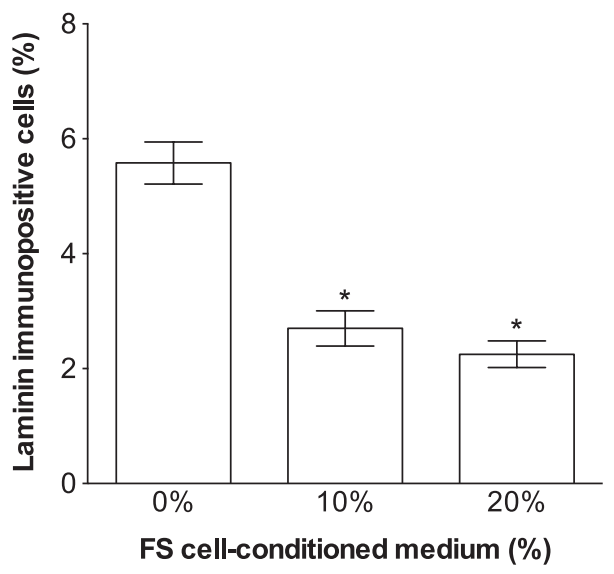

Fig. 6. Percentage of laminin-immunopositive cells in FS cell-deficient aggregates cultured with different concentrations of FS cell-conditioned medium ( $\mathrm{n}=10$, mean $\pm \mathrm{SEM}$ ). The number of laminin-immunopositive cells was counted and normalized by the total number of cells (DAPI). The proportion of laminin-immunopositive cells decreased when cultured in FS cell-conditioned medium. The proportion of lamininimmunopositive cells in cell aggregates cultured with normal medium $(0 \%)$ was significantly different from that in cells cultured with FS cellconditioned medium $(* \mathrm{p}<0.05$, Dunnett's test $)$.

\section{Discussion}

This study of 3D cell cultures of rat anterior pituitary cells revealed the importance of FS cells in the formation of cell aggregates, laminin release from gonadotrophs, and extracellular deposition of laminin. We also found that a humoral factor secreted from FS cells promoted laminin release from gonadotrophs.

Recently, we developed a 3D cell culture that utilizes a hanging drop method and showed that FS cells formed a 3D meshwork by interconnecting with other FS cells and that laminin formed string-like ramifying fibers in the extracellular space during cell aggregate formation [21]. Because cell aggregates became more regularly shaped with the concomitant development of the FS cell meshwork and laminin deposition, it is likely that this architecture is important in supporting the structure of the anterior pituitary. The present study examined the involvement of the FS cell meshwork and laminin deposition in cell aggregate formation. First, to test whether the FS cell meshwork is required for cell aggregate formation, we cultured anterior pituitary cells in the presence or absence of FS cells. As shown in Figure 1, amorphous cell aggregates were formed in the absence of FS cells, which contrasted with the formation of round/oval cell aggregates in the presence of FS cells. The results thus suggest that FS cells are important for cell aggregate formation and that the lack of the FS cell meshwork is a causative factor in the amorphous cell aggregates that formed in the absence of FS cells.

We then used immunohistochemical techniques to investigate whether laminin is deposited in FS cell-deficient 
cell aggregates. Intriguingly, laminin was not deposited in the extracellular space in the absence of FS cells (Fig. 1). Although there have been several studies on the effects of laminin on FS cells [7, 9-11, 19], this is the first report of an inverse effect, namely, that FS cells are required for laminin deposition.

Furthermore, we observed laminin-immunopositive cells in FS cell-deficient cell aggregates, and the proportion of laminin-immunopositive cells decreased as the number of FS cells increased (Figs. 1, 2). We speculated that laminin molecules accumulated within cells cultured without FS cells. Our previous research showed that laminin was produced by gonadotrophs and vascular endothelial cells [16]. We therefore performed double-immunostaining to identify laminin-immunopositive cells. Laminin-immunopositive cells were co-stained with LH $\beta$ only (Fig. 3) and were thus gonadotrophs rather than vascular endothelial cells.

In the next experiment, we investigated why the removal of FS cells causes laminin accumulation in gonadotrophs. The results indicated that laminin accumulation was caused by increased laminin synthesis in gonadotrophs and/or decreased laminin release from gonadotrophs. To test whether removal of FS cells increases laminin synthesis, we measured laminin mRNAs by real-time PCR. However, mRNA level was unchanged by removal of FS cells (Fig. 4), suggesting that removal of FS cells did not interfere with mRNA synthesis of laminin in gonadotrophs. To examine the effect on laminin release from gonadotrophs, we tested the hypothesis that a humoral factor and/or direct cell-cell contact are required for laminin release from gonadotrophs. We used FS cell-conditioned medium to test whether a humoral factor promotes laminin release. The results showed (Fig. 5) that FS cell-conditioned medium decreased the proportion of laminin-immunopositive cells, which suggests that a humoral factor contained in the FS cell-conditioned medium is required for laminin release from gonadotrophs. However, an additional increase in the proportion of FS cell-conditioned medium (from $10 \%$ to $20 \%$ ) did not further decrease the proportion of lamininimmunopositive cells. Therefore, the possibility that direct cell-cell contact between FS cells and gonadotrophs is also involved in laminin release cannot be excluded. Identification of the humoral factor will be necessary in order to understand in detail the mechanism underlying FS celldependent laminin release.

Interestingly, this study showed that the string-like ramifying structure of laminin deposition, which is normally observed in aggregates containing FS cells, was not reproduced by addition of FS cell-conditioned medium (Figs. 5, 6). This finding suggests that factors other than a humoral factor are required for laminin deposition. In general, a single laminin molecule is deposited after selfassembly [5, 23]. Although the mechanism of laminin selfassembly is not fully understood, Colognato and Yurchenco [1] reported that integrins, that is, laminin receptors, are required for laminin self-assembly. Our previous study showed that FS cells expressed several integrins [9]. Thus loss of contact with integrins might impair laminin deposition in FS cell-deficient cell aggregates. Therefore, FS cells might be important in extracellular laminin self-assembly and in laminin release from gonadotrophs.

Our previous study showed that vascular endothelial cells produce other laminin isoforms in the anterior pituitary gland [16]. However, unlike gonadotrophs, vascular endothelial cells of FS cell-deficient cell aggregates did not display cytoplasmic accumulation of laminin (Fig. 3). In addition, the mRNA level of the laminin isoform expressed in vascular endothelial cells (the $\alpha 4$ chain) was not altered by removal of FS cells (data not shown). Therefore, it appears that FS cells do not influence laminin synthesis or release from vascular endothelial cells.

In conclusion, our results suggest a novel function of FS cells: that they regulate laminin release from gonadotrophs through a humoral factor and subsequent laminin assembly. Because a variety of ECM components other than laminin are present in the anterior pituitary gland, future studies should examine whether FS cells are involved in regulating other ECM components, such as collagens.

\section{Declaration of Interest}

The authors have no conflict of interest that might prejudice the impartiality of this research.

\section{Acknowledgments}

We wish to thank Prof. K. Inoue (Saitama University, Japan) for supplying the transgenic rats, Prof. Y. Hanazono and Dr. T. Abe (Jichi Medical University) for performing fluorescence-activated cell sorting, and David Kipler, ELS (Supernatant Communications) for revising the language of the manuscript.

This work was partly supported by a Grant-in-Aid for Scientific Research (C) (22590192) and by a Grant-in-Aid for Young Scientists (B) (23790233) (25860147) from the Ministry of Education, Culture, Sports, Science and Technology of Japan, by promotional funds from the Keirin Race of the Japan Keirin Association, and by the Jichi Medical University Young Investigator Award from Jichi Medical University School of Medicine.

\section{References}

1. Colognato, H. and Yurchenco, P. D. (2000) Form and function: the laminin family of heterotrimers. Dev. Dyn. 218; 213-234.

2. Debbage, P. L., Griebel, J., Ried, M., Gneiting, T., DeVries, A. and Hutzler, P. (1998) Lectin intravital perfusion studies in tumor-bearing mice: micrometer-resolution, wide-area mapping of microvascular labeling, distinguishing efficiently and inefficiently perfused microregions in the tumor. J. Histochem. Cytochem. 46; 627-639.

3. Denef, C. (2008) Paracrinicity: the story of 30 years of cellular pituitary crosstalk. J. Neuroendocrinol. 20; 1-70. 
4. Devnath, S. and Inoue, K. (2008) An insight to pituitary folliculo-stellate cells. J. Neuroendocrinol. 20; 687-691.

5. Durbeej, M. (2010) Laminins. Cell Tissue Res. 339; 259-268.

6. Holck, S., Albrechtsen, R. and Wewer, U. M. (1987) Laminin in the anterior pituitary gland of the rat. Laminin in the gonadotrophic cells correlates with their functional state. Lab. Invest. 56 ; 481-488.

7. Horiguchi, K., Fujiwara, K., Ilmiawati, C., Kikuchi, M., Tsukada, T., Kouki, T. and Yashiro, T. (2011) Caveolin 3-mediated integrin $\beta 1$ signaling is required for the proliferation of folliculostellate cells in rat anterior pituitary gland under the influence of extracellular matrix. J. Endocrinol. 210; 29-36.

8. Horiguchi, K., Fujiwara, K., Kouki, T., Kikuchi, M. and Yashiro, T. (2008) Immunohistochemistry of connexin 43 throughout anterior pituitary gland in a transgenic rat with green fluorescent protein-expressing folliculo-stellate cells. Anat. Sci. Int. 83; 256260.

9. Horiguchi, K., Kikuchi, M., Kusumoto, K., Fujiwara, K., Kouki, T., Kawanishi, K. and Yashiro, T. (2010) Living-cell imaging of transgenic rat anterior pituitary cells in primary culture reveals novel characteristics of folliculo-stellate cells. J. Endocrinol. $204 ; 115-123$

10. Horiguchi, K., Kouki, T., Fujiwara, K., Kikuchi, M. and Yashiro, T. (2011) The extracellular matrix component laminin promotes gap junction formation in the rat anterior pituitary gland. $J$. Endocrinol. 208; 225-232.

11. Horiguchi, K., Ilmiawati, C., Fujiwara, K., Tsukada, T., Kikuchi, M. and Yashiro, T. (2012) Expression of chemokine CXCL12 and its receptor CXCR4 in folliculostellate (FS) cells of the rat anterior pituitary gland: the CXCL12/CXCR4 axis induces interconnection of FS cells. Endocrinology 153; 1717-1724.

12. Inoue, K., Couch, E. F., Takano, K. and Ogawa, S. (1999) The structure and function of folliculo-stellate cells in the anterior pituitary gland. Arch. Histol. Cytol. 62; 205-218.

13. Itakura, E., Odaira, K., Yokoyama, K., Osuna, M., Hara, T. and Inoue, K. (2007) Generation of transgenic rats expressing green fluorescent protein in S-100 $\beta$-producing pituitary folliculostellate cells and brain astrocytes. Endocrinology 148; 1518 1523.

14. Noda, T., Kaidzu, S., Kikuchi, M. and Yashiro, T. (2001) Topographic affinities of hormone-producing cells in the rat anterior pituitary gland. Acta Histochem. Cytochem. 34; 313-
319.

15. Paez-Pereda, M., Kuchenbauer, F., Arzt, E. and Stalla, G. K. (2005) Regulation of pituitary hormones and cell proliferation by components of the extracellular matrix. Braz. J. Med. Biol. Res. 38; 1487-1494.

16. Ramadhani, D., Tsukada, T., Fujiwara, K., Horiguchi, K., Kikuchi, M. and Yashiro, T. (2012) Laminin isoforms and laminin-producing cells in rat anterior pituitary. Acta Histochem. Cytochem. 45; 309-315.

17. Shirasawa, N., Mabuchi, Y., Sakuma, E., Horiuchi, O., Yashiro, T., Kikuchi, M., Hashimoto, Y., Tsuruo, Y., Herbert, D. C. and Soji, T. (2004) Intercellular communication within the rat anterior pituitary gland: X. Immunohistocytochemistry of S-100 and connexin 43 of folliculo-stellate cells in the rat anterior pituitary gland. Anat. Rec. A. Discov. Mol. Cell. Evol. Biol. 278; $462-473$.

18. Soji, T. and Herbert, D. C. (1989) Intercellular communication between rat anterior pituitary cells. Anat. Rec. 224; 523-533.

19. Syaidah, R., Horiguchi, K., Fujiwara, K., Tsukada, T., Kikuchi, M. and Yashiro, T. (2013) Laminin and collagen modulate expression of the small leucine-rich proteoglycan fibromodulin in rat anterior pituitary gland. Cell Tissue Res. 354; 633-638.

20. Timpl, R., Rohde, H., Robey, P. G., Rennard, S. I., Foidart, J. M. and Martin, G. R. (1979) Laminin-A glycoprotein from basement membranes. J. Biol. Chem. 254; 9933-9937.

21. Tsukada, T., Kouki, T., Fujiwara, K., Ramadhani, D., Horiguchi, K., Kikuchi, M. and Yashiro, T. (2013) Reassembly of anterior pituitary organization by hanging drop three-dimensional cell culture. Acta Histochem. Cytochem. 46; 121-127.

22. Tzu, J. and Marinkovich, M. P. (2008) Bridging structure with function: structural, regulatory, and developmental role of laminins. Int. J. Biochem. Cell Biol. 40; 199-214.

23. Yurchenco, P. D., Quan, Y., Colognato, H., Mathus, T., Harrison, D., Yamada, Y. and O'Rear, J. J. (1997) The alpha chain of laminin-1 is independently secreted and drives secretion of its beta- and gamma-chain partners. Proc. Natl. Acad. Sci. U S A 94; 10189-10194.

This is an open access article distributed under the Creative Commons Attribution License, which permits unrestricted use, distribution, and reproduction in any medium, provided the original work is properly cited. 